Submitted to ApJ

Preprint typeset using $\mathrm{AT}_{\mathrm{E}} \mathrm{X}$ style emulateapj v. 11/10/09

\title{
THE STELLAR-SUBHALO MASS RELATION OF SATELLITE GALAXIES
}

\author{
A. Rodríguez-Puebla, N. Drory and V. Avila-Reese \\ Instituto de Astronomía, Universidad Nacional Autónoma de México, A. P. 70-264, 04510, México, D.F., México. \\ Submitted to ApJ
}

\begin{abstract}
We extend the abundance matching technique (AMT) to infer the satellite-subhalo and centralhalo mass relations (MRs) of local galaxies, as well as the corresponding satellite conditional mass functions. We use the observed galaxy stellar mass function (GSMF) decomposed into centrals and satellites and the $\Lambda$-CDM distinct halo and subhalo mass functions as inputs. We explore the effects of defining the subhalo mass, $m_{\mathrm{sub}}$, at the time of (sub)halo accretion $\left(m_{\mathrm{sub}}^{\mathrm{acc}}\right)$ versus defining it at the time of observation $\left(m_{\mathrm{sub}}^{\mathrm{obs}}\right)$; and we test the standard assumption that centrals and satellites follow the same MRs. We show that this assumption leads to predictions in disagreement with observations, specially when $m_{\mathrm{sub}}^{\mathrm{obs}}$ is used. Instead, we find that when the satellite-subhalo MRs are constrained by the satellite GSMF, they are always different from the central-halo MR: the smaller the stellar mass, the less massive is the subhalo of satellites as compared to the halo of centrals of the same stellar mass. This difference is more dramatic when $m_{\mathrm{sub}}^{\mathrm{obs}}$ is used instead of $m_{\mathrm{sub}}^{\mathrm{acc}}$. On average, for stellar masses lower than $\sim 2 \times 10^{11} \mathrm{M}_{\odot}$, the dark mass of satellites decreased by $60-65 \%$ with respect to their masses at accretion time. We find that MRs for both definitions of subhalo mass yield satellite conditional mass functions (CSMF) in agreement with observations. Also, when these MRs are used in a halo occupation model, the predicted two-point correlation functions at different stellar mass bins agree with observations. The average stellar-halo MR is close to the MR of central galaxies alone, and conceptually this average MR is equivalent to abundance matching the cumulative total GSMF to the halo + subhalo mass function (the standard AMT). We show that the use of $m_{\mathrm{sub}}^{\text {obs }}$ leads to less uncertain MRs than $m_{\mathrm{sub}}^{\mathrm{acc}}$, and discuss some implications of the obtained satellite-subhalo MR. For example, we show that the tension between abundance and dynamics of Milky-Way satellites in the $\Lambda$-CDM cosmogony gives if the faint-end slope of the GSMF upturns to a value of $\sim-1.6$.

Subject headings: galaxies: abundances — galaxies: evolution — galaxies: halos — galaxies: luminosity function, mass function - galaxies: statistics - cosmology: dark matter.
\end{abstract}

\section{INTRODUCTION}

In recent years the abundance matching technique (AMT) has emerged as a simple yet powerful statistical approach for connecting galaxies to halos without requiring knowledge of the underlying physics (e.g., Vale \& Ostriker 2004; Kravtsov et al. 2004; Conrov. Wechsler \& Kravtsov 2006; Shankar et al.|2006; Baldry, Glazebrook \& Driver 2008; Conrov \& Wechsler 2009; Drory et al. 2009; Behroozi. Conroy \& Wechsler 2010, and references therein).

Briefly, the AMT assumes a one-to-one monotonic relationship between stellar and halo masses which can be constrained by matching the cumulative observed galaxy stellar mass function (GSMF) to the theoretical halo plus subhalo cumulative mass function. Interestingly enough, this simple approach successfully reproduces the observed spatial clustering of galaxies (e.g., Conroy, Wechsler \& Kravtsov 2006; Moster et al. 2010). The AMT allows to probe the average galaxy stellar-halo mass relation, $M_{*}\left(M_{\mathrm{h}}\right)$ (hereafter SHMR), delivering very useful information for constraining models of galaxy evolution (e.g., Guo et al. 2010; Firmani, Avila-Reese \& Rodríguez-Puebla 2010; Avila-Reese et al. 2011).

The above has motivated several authors to use the AMT extensively. For example, with the advent of

apuebla@astro.unam.mx large galaxy surveys at different redshifts, the AMT has been applied for constraining the evolution of the average SHMR (e.g., Conrov. Wechsler \& Kravtsov 2006; Drory et al. 2009; Conroy \& Wechsler 2009; Moster et al. 2010; Behroozi. Conroy \& Wechsler 2010). As a natural extension, these studies have been combined with predicted average halo mass aggregation histories in order to infer average galaxy $M_{*}$ growth histories as a function of mass (Conroy \& Wechsler 2009; Firmani \& Avila-Reese 2010, see for a review Avila-Reese \& Firmani 2011, and references therein). By including observational information on the gas content of galaxies, the AMT has been also used to constrain the baryon mass to $M_{\mathrm{h}}$ relation of galaxies (Baldrv. Glazebrook \& Driver 2008; Rodríguez-Puebla et al. 2011). Finally, variants of the AMT, where instead of mass functions, circular velocity functions or functions of any other galaxy/halo global property are employed, have been explored, too (e.g., Conrov. Wechsler \& Kravtsov 2006; Blanton, Geha \& West 2008; Trujillo-Gomez et al. 2011).

The AMT has been commonly applied to the total (central plus satellite galaxies) GSMF matched against the total (distinct plus satellite) halo population. This approach has been criticized, because quite different average SHMRs are obtained for different proposed forms of the satellite stellar-subhalo mass relation 
(SSMR, $\left.m_{*}\left(m_{\text {sub }}\right)\right)$ and the central SHMR $\left(M_{*}\left(M_{\mathrm{h}}\right)\right.$ ]; Neistein et al. 2011).

A common (questionable) assumption is that the SSMR is identical to the central SHMR. Under this assumption, it is also common to define subhalo mass at the time of accretion $\left(m_{\mathrm{sub}}^{\mathrm{acc}}\right)$ rather than at the time of observation $\left(m_{\mathrm{sub}}^{\mathrm{obs}}\right)$, when subhalos have lost a significant fraction of mass due to tidal stripping. The use of $m_{\mathrm{sub}}^{\text {acc }}$ has been justified because this way is avoided the question of subhalo mass loss, and regarding the satellite $m_{*}$, it is expected that it remains almost constant since its infall into the host halo. The projected twopoint correlation function of galaxies is reproduced under these assumptions (Conrov. Wechsler \& Kravtsov 2006; Moster et al. 2010). It should also be said that while the (local) SHMR for central galaxies has been determined (e.g., Mandelbaum et al. 2006; More et al. 2011), the stellar-subhalo mass relation for satellites/subhalos, SSMR, has not been yet discussed in detail in the literature.

In view of the above mentioned, some important questions arise. Why does using $m_{\mathrm{sub}}^{\mathrm{acc}}$ instead of $m_{\mathrm{sub}}^{\mathrm{obs}}$ lead to the correct clustering of galaxies? Does the $m_{\mathrm{sub}}^{\mathrm{acc}}-M_{*}$ relation reproduce the observed satellite GSMF, the conditional stellar mass function, and spatial clustering of galaxies at the same time? Even more fundamentally, if is not assumed that the SSMR is identical to the central SHMR, then, what follows for the SSMR, either using $m_{\text {sub }}^{\text {obs }} m_{\text {sub }}^{\text {acc }}$ ? Does it deviate from the central SHMR?

In this paper we extend the common AMT to constrain both the central SHMR and the SSMR separately, as well as the average (total) SHMR. By construction, this formalism also allows to predict the mean satellite conditional mass function (CSMF), i.e., the probability that satellites of a given stellar mass reside in distinct host halos of a given mass. We will (i) test whether the SSMR and the central SHMR follow the same shape; (ii) discuss the consequences of defining the subhalo mass at accretion time vs. at observed (present) time; and (iii) check the self-consistency of our predicted present-day central SHMR and SSMR by comparison with the observed satellite CSMF and the spatial clustering of galaxies.

This paper is laid out as follows. In Section 2 we present the AMT, focusing on the details of our extended abundance matching. In Section 3 we present the predicted stellar-halo mass relations $(\S \S 3.1)$ and satellite CSMFs $(\S \S 3.2)$ for cases when the SSMR is assumed equal to the central SHMR, and when both mass relations are independently constrained. In $\S \S 3.3$, a Halo Occupation Distribution (HOD) model is used to explore whether the predicted central SHMR's and SSMR's are consistent with the observed spatial clustering of galaxies. Section 4 is devoted to our conclusions and a discussion of the results and their implications.

All our calculations are based on a flat $\Lambda \mathrm{CDM}$ cosmology with $\Omega_{\Lambda}=0.73, h=0.7$, and $\sigma_{8}=0.84$.

\section{THE ABUNDANCE MATCHING TECHNIQUE}

\footnotetext{
${ }^{1}$ In order to make the distinction explicit, we shall use uppercase letters for the central galaxy and the distinct halo masses and lower-case letters for the satellite galaxy and subhalo masses.
}

In this section we describe the technique of matching abundances between central galaxies and halos and satellite galaxies and subhalos, separetely, which we present here as an extension to the standard AMT.

\subsection{Modeling the central \& satellite GSMFs}

To model the central GSMF, let $P_{\text {cen }}\left(M_{*} \mid M_{\mathrm{h}}\right)$ denotes the probability distribution function that a distinct halo of mass $M_{\mathrm{h}}$ hosts a central galaxy of stellar mass $M_{*}$. Then the number density of central galaxies with stellar masses between $M_{*}$ and $M_{*}+d M_{*}$ is given by

$$
\phi_{\mathrm{cen}}\left(M_{*}\right) d M_{*}=d M_{*} \int_{0}^{\infty} P_{\mathrm{cen}}\left(M_{*} \mid M_{\mathrm{h}}\right) \phi_{\mathrm{h}}\left(M_{\mathrm{h}}\right) d M_{\mathrm{h}} .
$$

For the population of satellite galaxies in individual subhalos, let $P_{\text {sat }}\left(m_{*} \mid m_{\text {sub }}\right)$ be the probability distribution function that a subhalo $m_{\text {sul }}{ }^{2}$ hosts a satellite galaxy of stellar mass $m_{*}$. Thus the average satellite CSMF (the number of satellite galaxies of stellar mass between $m_{*}$ and $m_{*}+d m_{*}$ that reside in distinct host halos of mass $M_{\mathrm{h}}$, e.g., Yang. Mo. \& van den Bosch 2009b) is

$\Phi_{s}\left(m_{*} \mid M_{\mathrm{h}}\right) d m_{*}=d m_{*} \int_{0}^{\infty} P_{\mathrm{sat}}\left(m_{*} \mid m_{\mathrm{sub}}\right) \Phi_{\mathrm{sub}}\left(m_{\mathrm{sub}} \mid M_{\mathrm{h}}\right) d m_{\mathrm{sub}}$

where $\Phi_{\text {sub }}\left(m_{\text {sub }} \mid M_{\mathrm{h}}\right)$ is the subhalo conditional mass function (subhCMF, i.e., the number of subhalos of mass between $m_{\text {sub }}$ and $m_{\text {sub }}+d m_{\text {sub }}$ residing in host halos of mass $M_{\mathrm{h}}$; e.g., Boylan-Kolchin et al. 2010). A natural link between the satellite GSMF, $\phi_{\text {sat }}$, and the distinct halo mass function $\left(\mathrm{HMF}, \phi_{\mathrm{h}}\right)$ arises once the satellite CSMF is given:

$$
\phi_{\mathrm{sat}}\left(m_{*}\right) d m_{*}=d m_{*} \int_{0}^{\infty} \Phi_{s}\left(m_{*} \mid M_{\mathrm{h}}\right) \phi_{\mathrm{h}}\left(M_{\mathrm{h}}\right) d M_{\mathrm{h}} .
$$

Inserting equation (2) into equation (3) and rearranging terms, the satellite GSMF can be rewritten in terms of $P_{\mathrm{sat}}\left(m_{*} \mid m_{\mathrm{sub}}\right)$ :

$\phi_{\mathrm{sat}}\left(m_{*}\right) d m_{*}=d m_{*} \int_{0}^{\infty} P_{\mathrm{sat}}\left(m_{*} \mid m_{\mathrm{sub}}\right) \phi_{\mathrm{sub}}\left(m_{\mathrm{sub}}\right) d m_{\mathrm{sub}}$,

where the subhalo mass function (subHMF) is given by

$\phi_{\mathrm{sub}}\left(m_{\mathrm{sub}}\right) d m_{\mathrm{sub}}=d m_{\mathrm{sub}} \int_{0}^{\infty} \Phi_{\mathrm{sub}}\left(m_{\mathrm{sub}} \mid M_{\mathrm{h}}\right) \phi_{\mathrm{h}}\left(M_{\mathrm{h}}\right) d M_{\mathrm{h}}$.

Equations (11) and (4) describe the abundance matching in its differential form for the central-halo and satellitesubhalo populations, respectively. The distribution probability $P_{\text {cen }}\left(M_{*} \mid M_{\mathrm{h}}\right)$ is defined by the mean $M_{*}\left(M_{\mathrm{h}}\right)$ relation and a scatter around it of $\sigma_{c}$, while the distribution probability $P_{\text {sat }}\left(m_{*} \mid m_{\mathrm{sub}}\right)$, assumed to be independent of host halo mass, is defined by the mean $m_{*}\left(m_{\mathrm{sub}}\right)$ relation and a scatter around it of $\sigma_{s}$. Observe that once $P_{\text {sat }}\left(m_{*} \mid m_{\text {sub }}\right)$ is given, the satellite CSMF is a prediction according to equation (2).

Here, $P_{\text {cen }}\left(M_{*} \mid M_{\mathrm{h}}\right)$ and $P_{\text {sat }}\left(m_{*} \mid m_{\text {sub }}\right)$ are modeled as lognormal distributions with a width (scatter around

2 Whenever we use $m_{\text {sub }}$ we refer to subhalo mass generically. In practice, that can either be the mass at accretion time, $m_{\mathrm{sub}}^{\mathrm{acc}}$, or at observation (present-day) time, $m_{\text {sub }}^{\text {obs }}$. 
the stellar mass) assumed to be constant and the same for both centrals and satellites, $\sigma_{c}=\sigma_{s}=0.173$ dex. Such a value was inferred for central galaxies from the analysis of general large group catalogs (YMB09) and it is supported by recent studies on the kinematics of satellite galaxies (More et al. 2011). Regarding the intrinsic scatter of the satellite-subhalo relation, it has not been discussed in detail in the literature. While the exploration of this scatter is beyond the scope of the present paper, our conclusions will not depend critically on the assumed value for it or even if it is allowed to depend on host halo mass. We will further discuss this question in $\$ 4.2$. Both $m_{*}\left(m_{\mathrm{sub}}\right)$ and $M_{*}\left(M_{\mathrm{h}}\right)$ are parametrized by the same modified two-power-law form proposed in Behroozi, Conroy \& Wechsler (2010). This five-parameters function is quite general and, in the context of the AMT, has been shown to reproduce the main features of a Schechter-like GSMF.

\subsection{The relation to standard abundance matching}

In the standard AMT the cumulative halo+subhalo mass function and the total observed cumulative GSMF are matched to determine the mass relation between halos and galaxies, which is assumed to be monotonic. In this context, no intrinsic scatter in the stellar mass at a given halo is assumed. In our approach, where the galaxy and halo populations are separated into centrals/satellites and distinct halo/subhalos, the latter entails that the probability distribution functions of centrals and satellites take the particular forms: $P_{\text {cen }}\left(\mathcal{M} \mid M_{\mathrm{h}}\right)=\delta\left(\mathcal{M}-M_{*}\left(M_{\mathrm{h}}\right)\right)$ and $P_{\text {sat }}\left(\mathcal{M} \mid m_{\text {sub }}\right)=$ $\delta\left(\mathcal{M}-m_{*}\left(m_{\text {sub }}\right)\right)$, where $M_{*}\left(M_{\mathrm{h}}\right)$ and $m_{*}\left(m_{\text {sub }}\right)$ are the mean central-halo and satellite-subhalo mass relations, and $\delta$ is for the $\delta$-Dirac function. The above "no scatter" probability distribution function for centrals applied in Eq. (1) would lead us to conclude that the cumulative central GSMF, $n_{\text {cen }}\left(>M_{*}\right)$, should match the cumulative distinct halo mass function, $n_{\mathrm{h}}\left(>M_{\mathrm{h}}\left(M_{*}\right)\right)$. The same reasoning applies for satellites/subhalos. Therefore, we arrive to the standard AMT formulation:

$$
n_{g}\left(>M_{*}\right)=n_{h}\left(>M_{\mathrm{h}}\right)+n_{\mathrm{sub}}\left(>M_{\mathrm{h}}\right),
$$

where $n_{g}\left(>M_{*}\right) \equiv n_{\text {cen }}\left(>M_{*}\right)+n_{\text {sat }}\left(>M_{*}\right)$ is the total GSMF.

Since the abundance matching can be applied to centrals/halos and satellites/subhalos separately, let analyze now only the latter. Under the assumption that the $m_{*}\left(m_{\text {sub }}\right)$ relation is independent of the host halo mass, it is clear that using either the abundance matching of all satellites and all subhalos, $n_{\text {sat }}\left(>m_{*}\right)=n_{\text {sub }}\left(>m_{\text {sub }}\right)$, or the matching of their corresponding mean occupational numbers, one may find exactly the same $m_{*}\left(m_{\mathrm{sub}}\right)$ relation. In this sense, we state that matching abundances is equivalent to matching occupational numbers:

$$
\begin{array}{r}
\left\langle N_{s}\left(>m_{*} \mid M_{\mathrm{h}}\right)\right\rangle=\left\langle N_{\mathrm{sub}}\left(>m_{\mathrm{sub}} \mid M_{\mathrm{h}}\right)\right\rangle \\
\Longleftrightarrow n_{\text {sat }}\left(>m_{*}\right)=n_{\text {sub }}\left(>m_{\mathrm{sub}}\right) .
\end{array}
$$

In the case that the probability distribution function $P_{\text {sat }}\left(m_{*} \mid m_{\text {sub }}\right)$ includes scatter around the mean SSMR, as we consider here, the above conclusion remains the same whilst $P_{\text {sat }}\left(m_{*} \mid m_{\text {sub }}\right)$ is assumed to be independent on halo mass. In general, the inclusion of constant scatter in the galaxy-halo mass relations is not a conceptual problem for the AMT, but it slightly modifies the shape of the mass relations at the high mass end (see Behroozi, Conroy \& Wechsler 2010). Finally, note that if $P_{\text {sat }}\left(m_{*} \mid m_{\text {sub }}\right)$ depends on $M_{\mathrm{h}}$, then $\phi_{\text {sat }}$ may not be directly related to $\phi_{\text {sub }}$ (see Eq. 4) and using either the matching of satellites and subhalo abundances or the matching of their corresponding occupational numbers would not lead to find exactly the same $m_{*}\left(m_{\mathrm{sub}}\right)$.

\subsection{Inputs for matching abundances}

The inputs required for the procedure described above are the subhCMF, the distinct HMF, and the observed satellite and central GSMFs.

For the subhCMF, we use the results obtained in Boylan-Kolchin et al. (2010, BK10) based on the analysis of the Millennium-II Simulation. This is a re-simulation with the same resolution of a smaller volume of the Millennium Simulation. It consists of $2160^{3}$ particles, each of mass $m=6.885 \times 10^{6} h^{-1} \mathrm{M}_{\odot}$ in a periodic cube of length $L=100 h^{-1} \mathrm{Mpc}$. Observe that this mass particle resolution is around four orders of magnitude below the subhalo masses required $\left(\sim 10^{10} h^{-1} \mathrm{M}_{\odot}\right)$ to match the lower stellar mass limit in the YMB09 GSMF. The fitting formula for the cumulative subhCMF reported in BK10 at the $\left[10^{12}, 10^{12.5}\right] h^{-1} \mathrm{M}_{\odot}$ mass interval is:

$$
\left\langle N_{\mathrm{sub}}\left(>m_{\mathrm{sub}} \mid M_{\mathrm{h}}\right)\right\rangle=\mu_{0}\left(\frac{\mu}{\mu_{1}}\right)^{a} \exp \left[-\left(\frac{\mu}{\mu_{\mathrm{cut}}}\right)^{b}\right] \text {, }
$$

where $\mu=m_{\mathrm{sub}} / M_{\mathrm{h}}$. For $m_{\mathrm{sub}}=m_{\mathrm{sub}}^{\text {acc }}$, $\left(\mu_{0}, \mu_{1}, \mu_{\text {cut }}, a, b,\right)=(1,0.038,0.225,-0.935,0.75)$, while for $m_{\text {sub }}=m_{\text {sub }}^{\mathrm{obs}}$, $=$ $\left(1.15^{\left(\log M_{\mathrm{h}}-12.25\right)}, 0.01,0.096,-0.935,1.29\right)$. According to BK10, the shape of the $m_{\mathrm{sub}}^{\mathrm{obs}}$ subhCMF remains the same for other halo masses but its normalization, $\mu_{0}$, systematically increases with $M_{\mathrm{h}}$, roughly by $15 \%$ per dex in $M_{\mathrm{h}}$. Such a behavior has been reported in an analysis of the Millennium simulations by Gao et al. (2011, for closely related results, with small differences in the amplitude, see also Gao et al. 2004; van den Bosch. Tormen \& Giocoli 2005; Zentner et al. 2005; Angulo et al. 2009; Giocoli et al. 2010). we introduce the dependence $\mu_{0}=1.15^{\left(\log M_{\mathrm{h}}-12.25\right)}$, where $\mu_{0}=1$ at $\log M_{\mathrm{h}}=12.25$. In the case of the $m_{\mathrm{sub}}^{\mathrm{acc}}$ subhCMF, the normalization factor is nearly independent on $M_{\mathrm{h}}$, i.e., $\mu_{0}=1$ (BK10; see also Giocoli. Tormen \& van den Bosch 2008). The subhCMF is given by $\Phi_{\text {sub }}=d N_{\text {sub }} / d m_{\text {sub }}$.

In order to construct the $m_{\text {sub }}^{\text {acc }}$ subhCMF, BK10 traced each subhalo back in time so that they were able to find the point at which its bound mass reached a maximum, i.e., the time the halo became a subhalo. The latter guarantees that we are working with the surviving population of accreted halos and no further assumptions on the merging process are necessary.

The difference between the Millennium-II simulation cosmology and ours leads to differences in the resulting abundances of subhalos of roughly a few percent in the amplitude of the subhalo mass function (BK10). This is also supported by previous works that explored the impact of changing cosmological parameters on the subhalo occupational statistics (e.g., Zentner \& Bullock 
2003). Additionally, to be consistent with the same cosmology for which the subhalo subhCMF was inferred, we repeated all the analysis to be showed below but using the WMAP1 cosmology. We find that all our results are practically the same.

For the distinct HMF, we will use the formula given by Sheth \& Tormen (1999). This formula provides a reasonable fit to the the virial mass ${ }^{3}$ function at $z \sim 0$ measured in large cosmological N-body simulations (e.g., Klypin. Truilllo-Gomez \& Primack 2011; Cuesta et al. 2008).

For our purpose, the decomposition of the GSMF and the CSMFs into centrals and satellites galaxies is necessary. Using a large general group catalog (Yang et al. 2007) based on the data from the SDSS, YMB09 constructed and studied the decomposition of the GSMF and the CSMFs into centrals and satellites galaxies. In that paper, a central galaxy was defined as the most massive galaxy in a group and the remaining galaxies as satellites. For the mass completeness limit in the GSMF, they adopted the value as function of redshift proposed in van den Bosch et al. (2008). They have also taken into account incompleteness in the group mass by considering an empirical halo-mass completeness limit (for details we refer the reader to YMB09).

Where necessary, halo masses are converted to match our definition of virial mass and stellar masses are converted to the Chabrier (2003) IMF. In particular, YMB09 defined halo masses as being 180 times the background density, according to Giocoli et al. 2010, see their appendix B) these halos are $\sim 11 \%$ larger than our definition of virial mass.

\subsection{Procedure and uncertainties}

We constrain the parameters of the functions proposed to describe the central SHMR and SSMR by means of Eqs. (11) and (3), and by using the Powell's directions set method in multi-dimensions for the minimization (Press et al. 1992). Note that in our analysis the reported statistical errors in the GSMFs, as well as the intrinsic scatter in the mass relations are taken into account. However, we will not analyze rigorously here the effects of uncertainties on the mass relations as well as their errors. Instead, we remit the reader to previous works (Moster et al. 2010; Behroozi, Conroy \& Wechsler 2010; Rodríguez-Puebla et al.|2011).

Behroozi. Conrov \& Wechsler (2010) studied in detail the uncertainties and effects on the average SHMR due to different sources of error like those in the observed GSMFs, including stellar mass estimates; in the halo mass functions; in the uncertainty of the cosmological parameters; and in linking galaxies to halos, including the intrinsic scatter in this connection. These authors have found that the largest uncertainty by far in the SHMR is due to systematic shifts in the stellar estimates. The second important source of uncertainty is due to the intrinsic scatter, that we take into account in our analysis. Other statistical and sample variance errors have negligible effects, at least for local galaxies. According to the

\footnotetext{
3 The mass enclosed within the radius at which, according to the spherical collapse model, the overdensity of a sphere is $\Delta_{\text {vir }}$ times larger than the matter critical density of the used cosmological model; for the cosmology assumed here, $\Delta_{\mathrm{vir}}(z=0)=97$.
}

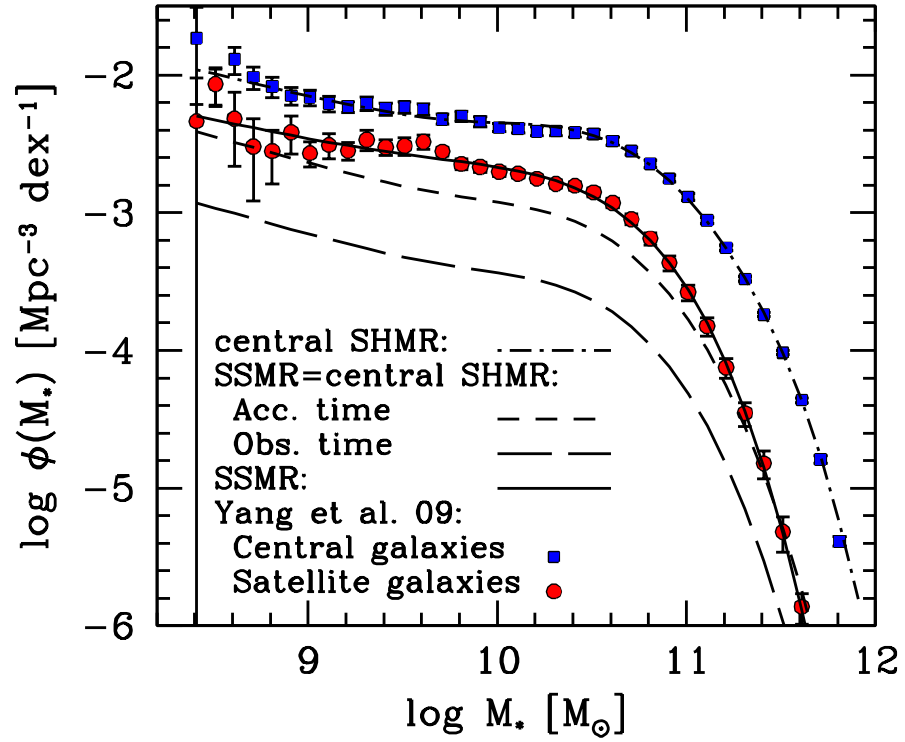

FIG. 1. - Satellite GSMFs calculated under the assumption that $P_{\text {sat }}\left(m_{*} \mid m_{\mathrm{sub}}\right)=P_{\mathrm{cen}}\left(M_{*} \mid M_{\mathrm{h}}\right)$ and for the cases $m_{\mathrm{sub}}^{\text {acc }}$ (long-

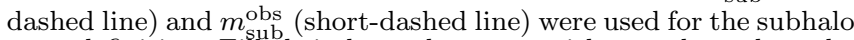
mass definition. Filled circles and squares with error bars show the YMB09 central and satellite GSMFs, respectively. The solid line is for the case when $P_{\text {sat }}\left(m_{*} \mid m_{\text {sub }}\right)$ was determined using the YMB09 satellite GSMF as a constraint, i.e., is the best model fit to this function.

Behroozi, Conroy \& Wechsler (2010) study, the statistical and systematical uncertainties account for $1 \sigma$ errors in the SHMR of approximately 0.25 dex at all masses, which is almost totally due to the uncertainty in stellar mass estimates. We have explored here also the effects of the subhalo CMF uncertainty on the SSMR. By using the $25 \%$ per dex in $M_{\mathrm{h}}$ variation reported by Giocoli et al. (2010) (instead of 15\%), we find that the SSMR shifts in $m_{*}$ by only $\approx 0.04$ dex.

\section{RESULTS}

\subsection{The satellite GSMF and the SSMR}

By means of the procedure described in Section 2, we calculate first the satellite GSMF (Fig. 1) when the SSMR and the central SHMR are assumed to be the same, i.e., $m_{*}\left(m_{\mathrm{sub}}\right)=M_{*}\left(M_{\mathrm{h}}\right)$. This is equivalent to assume that $P_{\text {sat }}\left(m_{*} \mid m_{\mathrm{sub}}\right)=P_{\text {cen }}\left(M_{*} \mid M_{\mathrm{h}}\right)$ if the intrinsic scatter of both relations is the same. We obtain the central SHMR by matching abundances of YMB09 central galaxies to distinct halos. This relation and the subhalo mass function obtained from the theoretical subhCMF (eq. 8), are used to infer the satellite GSMF (eq. 4). The satellite GSMF is presented for the two cases of subhalo mass definition: $\phi_{\text {sat,acc }}$ when $m_{\text {sub }}^{\text {acc }}$ is used (long-dashed line), and $\phi_{\text {sat,obs }}$ when $m_{\text {sub }}^{\text {obs }}$ is used (short-dashed line). The observational results of YMB09 are plotted as well.

Under the assumption that $m_{*}\left(m_{\mathrm{sub}}\right)=M_{*}\left(M_{\mathrm{h}}\right)$, the predicted number density of satellites at masses below the knee is underestimated on the average by a factor of $\sim 2$ when using $m_{\text {sub }}^{\text {acc }}$, and $\sim 5$ when using $m_{\text {sub }}^{\text {obs }}$. Note that the former is closer to the YMB09 data. The reason is simply because the normalization of the $m_{\text {sub }}^{\text {acc }}$ subHMF is higher and closer to the distinct HMF than the normalization of the $m_{\text {sub }}^{\text {obs }}$ subHMF. Therefore, satellites of 

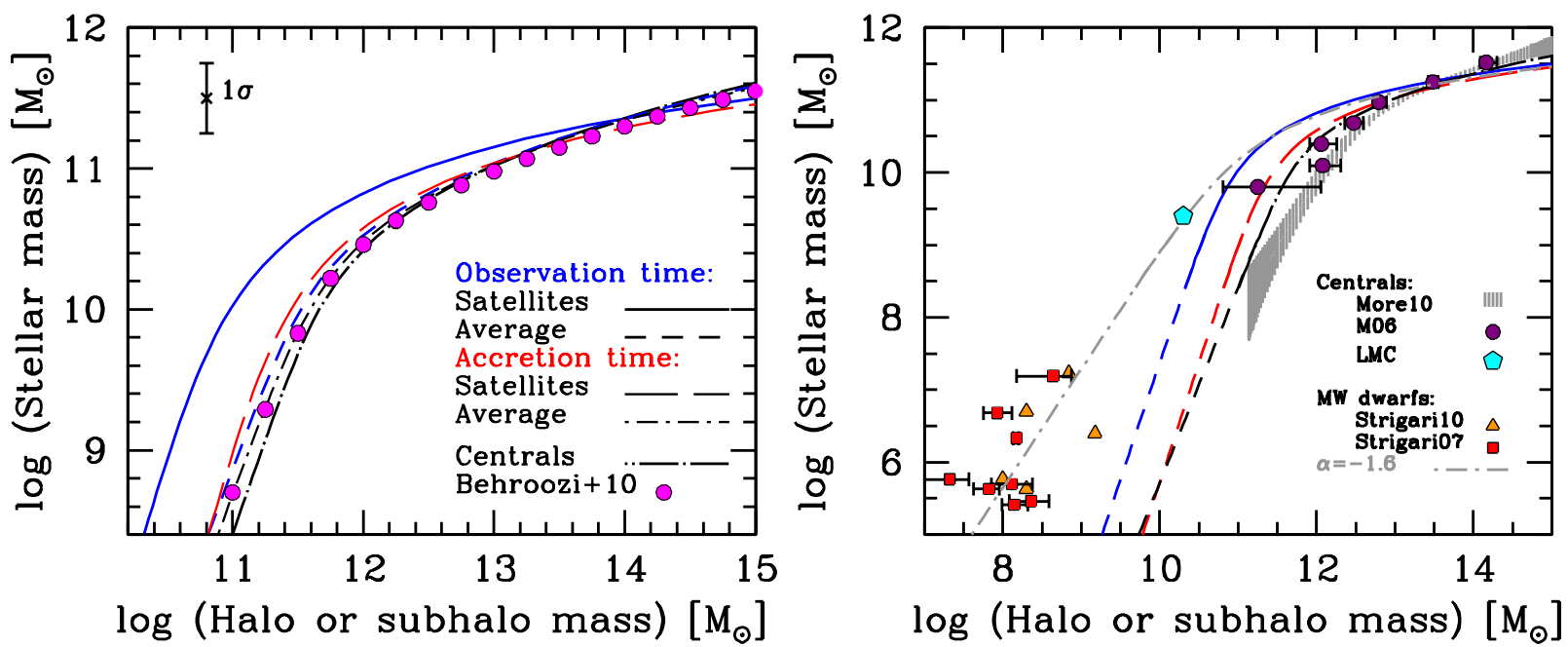

FIG. 2.- Left panel: Inferred mass relations for satellite galaxies when the subhalo mass is defined as $m_{\mathrm{sub}}^{\mathrm{obs}}$ (blue solid line) and as $m_{\mathrm{sub}}^{\text {acc }}$ (red long-dashed-line), and for central galaxies (black dot-long-dashed line). The density-weighted average relation when using subhalo mass $m_{\mathrm{sub}}^{\mathrm{obs}}$ is plotted with a blue short-dashed line, while when using subhalo mass $m_{\mathrm{sub}}^{\mathrm{acc}}$ is plotted with a gray short-dotted line. For comparison, the (average) mass relation obtained in Behroozi. Conrov \& Wechsler (2010) is also plotted (filled circles). Right panel: A comparison of the mass relations of satellite and central galaxies with direct observational inferences (the same line code of left panel for models is used; dashed lines indicate extrapolations to lower masses). Filled circles with error bars correspond to the mass relation of central galaxies from the analysis of staked weak-lensing in Mandelbaum et al. (2006). Dashed area indicates the $68 \%$ of confidence in the mass relation of central galaxies using the kinematics of satellites (More et al.|2011). The inferred total mass at the tidal radii for the brightest dwarf galaxies obtained in Strigari et al. (2007) and Strigari, Frenk \& White (2010) are plotted with filled squares and triangles, respectively. Filled pentagon shows the mass at the tidal radius for the Large-Magallanic Cloud (Weinberg 1999). The gray dotted-dashed curve is the $m_{\mathrm{sub}}^{\mathrm{obs}}$ SSMR assuming a faint-end slope in the satellite GSMF of $\alpha=-1.6$.

equal $m_{*}$ are expected to have a higher number density when using the accreted-time $\left(m_{\text {sub }}^{\text {acc }}\right)$ subHMF compared to using the observed-time ( $m_{\text {sub }}^{\text {obs }}$; present-day) subHMF.

However, neither $m_{\mathrm{sub}}^{\mathrm{acc}}$ nor $m_{\mathrm{sub}}^{\mathrm{obs}}$ are able to reproduce the observed satellite GSMF, and the discrepancy is due to the basic assumption of a common stellar mass(sub)halo mass relation for centrals and satellites. In the case that $m_{\mathrm{sub}}=m_{\mathrm{sub}}^{\mathrm{acc}}$, this is equivalent to assume that the SSMR is independent of redshift. But in fact this cannot be the case since the satellite mass $m_{*}$ hardly will remain the same since it was accreted to the present epoch. On the other hand, when using $m_{\text {sub }}=m_{\text {sub }}^{\text {obs }}$, that the SSMR is equal to the central SHMR implies that both have evolved, on average, identically. This cannot be the case because it is evident that the population of subhalos evolved differently to distinct halos, mainly by losing mass due to tidal striping (e.g., Kravtsov et al. 2004; van den Bosch, Tormen \& Giocoli 2005).

The next step in our analysis is to allow the SSMR and central SHMR to be different, i.e., $m_{*}\left(m_{\text {sub }}\right) \neq M_{*}\left(M_{\mathrm{h}}\right)$. In this case, $P_{\text {sat }}\left(m_{*} \mid m_{\text {sub }}\right)$ is determined by means of Eq. (4) using the YMB09 satellite GSMF as a constraint. For the subhCMF, we again use both definitions of subhalo mass, $m_{\text {sub }}^{\text {acc }}$ and $m_{\text {subs }}^{\text {obs }}$ For illustrative purpose, we present the resulting satellite GSMF for the case when $m_{\text {sub }}^{\text {obs }}$ was used (solid line in Fig 1] an almost identical GSMF is obtained when $m_{\text {sub }}^{\text {acc }}$ was used).

As shown in Fig. 2, the SSMRs obtained by using $m_{\text {sub }}^{\text {acc }}$

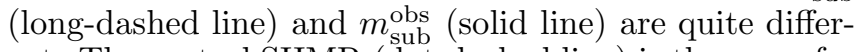
ent. The central SHMR (dot-dashed line) is the same for both cases. The error bar in the left panel shows a $1 \sigma$ uncertainty of 0.25 dex in the normalization of the mass relations. This is roughly the uncertainty estimated by Behroozi, Conroy \& Wechsler (2010) taking into account all the systematical and statistical sources of errors (see $\S \S 2.4)$.

When using the accretion-time subhalo mass, $m_{\text {sub }}^{\text {acc }}$, we note that the resulting SSMR at $\log \left(m_{*} / \mathrm{M}_{\odot}\right)<11$ systematically lies above the central SHMR, with differences in the stellar-mass axis (halo-mass axis) of $\sim 0.5 \mathrm{dex}(0.2$ dex) at the smallest masses. For $\log \left(M_{*} / \mathrm{M}_{\odot}\right)>11$ this trend is inverted, but the differences between central and satellites are very small. However, the relation obtained this way should be taken with caution. By construction, each $m_{\mathrm{sub}}^{\mathrm{acc}}$ is itself a cumulative distribution of all the objects accreted in a time interval $\Delta z$. Therefore such a SSMR entails that all accreted objects of mass $m_{\text {sub }}^{\text {acc }}$ would evolve, on average, to host the same $m_{*}$ despite having been accreted at different times. We discuss this in $\S \S 4.1$.

When using the observation-time (present-day) subhalo mass, $m_{\mathrm{sub}}^{\mathrm{obs}}$, the SSMR (solid blue line) and central SHMR are very different, though they show the same trend as when using $m_{\text {sub. }}^{\text {acc }}$ For example, on average, a satellite with $\log \left(M_{*} / \mathrm{M}_{\odot}\right)=10$ resides in a subhalo a factor of $\sim 4$ less massive than the halo of a central galaxy with the same stellar mass. Notice that $m_{\text {sub }}^{\text {obs }}\left(m_{*}\right)<m_{\text {sub }}^{\text {acc }}\left(m_{*}\right)$ and that the difference increases the lower the mass is. This is consistent with the picture that most massive subhalos, on average, fell into larger halos just very recently and they have not had time to lose significant amounts of mass due to tidal striping, in 
contrast to the lowest mass subhalos.

This also suggests that the SSMR for both definitions of subhalo mass should tend to the central SHMR at the high-mass end, but this is not the case as seen in Fig. 2 where small differences remain. The possible reasons are that, firstly, the intrinsic scatter around the stellar-(sub)halo mass relations is actually lower for the former than for the latter (here we assumed it to be the same for satellites and centrals, see §4.2). Secondly, that the YMB09 satellite GSMF may underestimate the true satellite mass function at large masses (see also Skibba et al. 2011).

Fiber collisions could introduce some systematic error that may affect the YMB09 group catalog. To study the impact of this possible systematic error, YMB09 divided their group catalog into two sample: one that uses galaxies with known redshifts, and another that includes galaxies that lack redshifts due to fiber collisions. When compared the corresponding satellite CSMFs from both samples (see their Fig. 6), they found that the sample for which the correction for fiber collisions has been taking into account, has a higher amplitude of the CSMFs than when this correction has not been applied, specially in low mass halos. However, the difference is very marginal and well within the error bars. We conclude that fiber collisions in the YMB09 group catalog are not a serious source of systematics able to affect our conclusions. Regarding completeness and contamination of their group catalog (for details see Yang et al. 2007), 80\% have a completeness greater than 0.6 , while $85 \%$ have a contamination lower than 0.5 . In terms of purity, their halobased group finder is consistent with the ideal situation.

Finally, we note that the mass relation usually obtained by matching abundances between the total GSMF and the halo plus subhalo mass function, in the light of the decomposition into centrals and satellites, could be interpreted as a density-weighted average SHMR:

$$
\left\langle M_{*}(M)\right\rangle_{\phi}=\frac{\phi_{\mathrm{sub}}(M)}{\phi_{\mathrm{DM}}(M)} m_{*}(M)+\frac{\phi_{\mathrm{h}}(M)}{\phi_{\mathrm{DM}}(M)} M_{*}(M),
$$

where $\phi_{\mathrm{DM}}(M)=\phi_{\mathrm{sub}}(M)+\phi_{\mathrm{h}}(M), m_{*}(M)$ is the mean SSMR and $M_{*}(M)$ is the mean central SHMR. This relation is plotted in Fig. 2 with short-dashed-dot and short-dashed lines when using $m_{\mathrm{sub}}^{\mathrm{acc}}$ and $m_{\mathrm{sub}}^{\mathrm{ob}}$, respectively. Since most galaxies in the YMB09 catalog are centrals, the central SHMR is very close to the densityweighted average SHMR. For comparison, we plotted the Behroozi. Conroy \& Wechsler (2010) average mass relation (filled circles), which is in excellent agreement with our density-weighted average SHMR when using the accreted-time subhalo mass, $m_{\text {sub }}^{\text {acc }}$.

Observe that differences between the satellite and the average (total) mass relations are small when $m_{\text {sub }}^{\text {acc }}$ is used, while differences become dramatic when $m_{\mathrm{sub}}^{\mathrm{obs}}$ is used. The above explains why under the assumption that $m_{*}\left(m_{\mathrm{sub}}^{\mathrm{acc}}\right)=M_{*}\left(M_{\mathrm{h}}\right)=\left\langle M_{*}(M)\right\rangle_{\phi}$, the resulting satellite GSMF are closer to observations. On the other hand, since the $m_{\text {sub }}^{\text {acc }}$ subHMF has a higher normalization than the $m_{\mathrm{sub}}^{\mathrm{obs}}$ subHMF, the above shows that when assuming $m_{*}\left(m_{\mathrm{sub}}^{\mathrm{obs}}\right)=M_{*}\left(M_{\mathrm{h}}\right)=\left\langle M_{*}(M)\right\rangle_{\phi}$, we should expect that the resulting satellite GSMF is significantly below the observed satellite GSMF.

\subsubsection{Comparison with other observational inferences}

In the right panel of Fig. 2] we plot some observational inferences of halo and subhalo masses as a function of stellar mass. The inferred $\left\langle M_{\mathrm{h}}\right\rangle\left(M_{*}\right)$ of central galaxies from staked weak-lensing studies using the SDSS (Mandelbaum et al. 2006) are shown as filled circles with error bars. Mandelbaum et al. (2006) reported the data actually for blue and red galaxies separately. We estimated the average mass relation for central galaxies as: $\left\langle M_{\mathrm{h}}\right\rangle\left(M_{*}\right)=f_{b}\left(M_{*}\right)\left\langle M_{\mathrm{h}}\right\rangle_{b}\left(M_{*}\right)+f_{r}\left(M_{*}\right)\left\langle M_{\mathrm{h}}\right\rangle_{r}\left(M_{*}\right)$, where $f_{b}\left(M_{*}\right)$ and $f_{r}\left(M_{*}\right)$ are the blue and red galaxy fractions in the sample, and $\left\langle M_{\mathrm{h}}\right\rangle_{b}$ and $\left\langle M_{\mathrm{h}}\right\rangle_{r}$ are the corresponding blue and red mass relations. The inferred $\left\langle\log \left(M_{*}\right)\right\rangle\left(M_{\mathrm{h}}\right)$ for central galaxies from staked kinematics of satellites (More et al. 2011) are plotted as the dashed area indicating the $68 \%$ of confidence.

Our inferred central SHMR (dotted-dashed curve) is consistent with the weak-lensing inferences at all masses, and with the satellite kinematics inferences at masses $M_{*} \gtrsim 10^{11} \mathrm{M}_{\odot}$; for smaller masses, our halo masses are a factor up to $\sim 2$ smaller than the satellite kinematics inferences. In fact, it was already noted that using the kinematics of satellite galaxies yields halo masses around low mass galaxies that are systematically larger than most other methods, specially for red central galaxies (More et al. 2011; Skibba et al. 2011; Rodríguez-Puebla et al. 2011).

Regarding satellites, unfortunately, there are not direct inferences of their subhalo masses. Some modeldependent estimates based on dynamical observations of Milky-Way (MW) satellites were presented in the literature. For example, using the line-of-sight velocity dispersions measured for the brightest spheroidal dwarf galaxies, Strigari et al. (2007) and Strigari. Frenk \& White (2010) determined their masses within their tidal radii. These dynamical masses, plotted in Fig. 2 (filled squares and triangles, respectively), are expected to be of the order of $m_{\mathrm{sub}}^{\mathrm{obs}}$. We also plot an estimate of the mass at the tidal radius for the Large-Magallanic Cloud (filled pentagon, Weinberg 1999). The SSMRs constrained here do not extend to the small masses of MW satellites but we plot their extrapolations to these masses (dashed curves). The gray dotted-dashed curve will be discussed in $\S \S 4.3$

\subsection{The satellite CSMF}

From the approach described in Section 2, another statistical quantity that deserves to be subject of study is the satellite CSMF (Eq.2). We calculate the mean halodensity-weighted CSMF at the $\left[M_{\mathrm{h}_{1}}, M_{\mathrm{h}_{2}}\right]$ bin as:

$$
\left\langle\Phi_{s}\right\rangle=\frac{\int_{M_{\mathrm{h}_{1}}}^{M_{\mathrm{h}_{2}}} \Phi_{s}\left(m_{*} \mid M_{\mathrm{h}}\right) \phi_{\mathrm{h}}\left(M_{\mathrm{h}}\right) d M_{\mathrm{h}}}{\int_{M_{\mathrm{h}_{1}}}^{M_{\mathrm{h}_{2}}} \phi_{\mathrm{h}}\left(M_{\mathrm{h}}\right) d M_{\mathrm{h}}} .
$$

This quantitiy has been inferred from observations by YMB09, again using their SDSS galaxy catalog (filled circles with error bars in Fig. 3).

First, we consider again the case assuming $m_{*}\left(m_{\text {sub }}\right)=$ $M_{*}\left(M_{\mathrm{h}}\right)$. When $m_{\text {sub }}$ is defined at the observation time, the resulting CSMFs are lower than the YMB09 CSMFs by a factor of $\sim 5$ in the power-law regime (roughly the same factor by which $\phi_{\text {sat,obs }}$ is lower than the YMB09 observed satellite GSMF). Similarly, when $m_{\text {sub }}$ is defined at the accretion time, the predicted CSMFs in 
the power-law regime are below the YMB09 CSMFs by nearly the same factor, $\sim 2$, that $\phi_{\text {sat, acc }}$ lies below the satellite GSMF. The normalization of the CSMF increases faster with $M_{\mathrm{h}}$ when $m_{\mathrm{sub}}^{\mathrm{obs}}$ is used instead of $m_{\text {sub }}^{\text {acc }}$. This is because we allow the $m_{\text {sub }}^{\text {obs }}$ subhCMF normalization to vary with host halo mass, while the $m_{\text {sub }}^{\text {acc }}$ subhCMF normalization is independent of host halo mass.

The black continuous ( $m_{\mathrm{sub}}^{\mathrm{obs}}$ ) and blue long-dashed $\left(m_{\text {sub }}^{\text {acc }}\right)$ lines in Fig. 3 (almost indistinguishable one from other) are the predictions when $P_{\text {sat }}\left(m_{*} \mid m_{\text {sub }}\right)$ has been constrained by means of the observed satellite GSMF. The agreement of the predicted satellite CSMF's, for both $m_{\text {sub }}^{\text {obs }}$ and $m_{\text {sub }}^{\text {acc }}$ with the YMB09 CSMF's is now remarkable at all halo mass bins for low/intermedium stellar masses. Although, as above, the normalization of the CSMF's increases faster when $m_{\text {sub }}=m_{\text {sub }}^{\text {obs }}$ than when $m_{\text {sub }}=m_{\text {sub }}^{\text {acc }}$, the differences between both cases at any mass are less than 0.05 dex, within the error bars of the observational data.

Despite the overall agreement, for halo mass bins lower than $\sim 10^{13} \mathrm{M}_{\odot}$, the number of massive satellite galaxies is overestimated, specially at the lowest $M_{\mathrm{h}}$ bins. A possible reason for this is the assumption that the scatter in $P_{\text {sat }}\left(m_{*} \mid m_{\text {sub }}\right)$ is constant while in reality it could depend on $M_{\mathrm{h}}$ as well as on $m_{\mathrm{sub}}$. However, the probability of finding massive satellite galaxies in halos less massive than $\sim 10^{13} \mathrm{M}_{\odot}$ is low and they do not contribute significantly to the mean total density of satellite galaxies. Therefore, this assumption does not change our conclusions, see also $\S 4$.

Our analysis shows that assuming $P_{\text {sat }}\left(m_{*} \mid m_{\text {sub }}\right)=$ $P_{\text {cen }}\left(M_{*} \mid M_{\mathrm{h}}\right)$ the resulting satellite CSMFs are not consistent with observations. Instead, when $P_{\text {sat }}\left(m_{*} \mid m_{\text {sub }}\right)$ is independently constrained using the observed satellite GSMF, there is a clear agreement, no matter what definition of $m_{\text {sub }}$ was employed for the subhCMF.

\subsection{Abundance matching and clustering}

It has been noted in the literature that the average (total) SHMR obtained with the standard AMT is consistent with the observed spatial clustering of galaxies (Conroy, Wechsler \& Kravtsov 2006; Moster et al. 2010). We will test now whether this is the case for the mass relations of central and satellite galaxies obtained here with our extended AMT. We will compute the galaxy projected correlation function by means of a HOD model for each of the mass relation obtained in $\S 3.1$.

A HOD model is a statistical tool mainly used to describe the clustering of galaxies (e.g. Berlind \& Weinberg 2002; Cooray \& Sheth 2002; Yang. Mo \& van den Bosch 2003; Zehavi et al. 2005, 2011; Leauthaud et al. 2011a,b; Yang et al. 2011, and more references therein). In contrast to the AMT, which is a quasi empirical tool, a HOD employs modeling motivated by results of cosmological $N$-body (e.g., Kravtsov et al. 2004) and hydrodynamical (e.g., Zheng et al. 2005) simulations.

In short, a HOD model describes the probability that a halo of mass $M_{\mathrm{h}}$ hosts a number of $N$ galaxies with stellar masses greater than $M_{*}$. Once the occupational numbers are defined, the two-point correlation function can be computed assuming that the total mean number of galaxy pairs is the contribution of all pairs coming from galaxies in the same halo (one-halo term) and pairs from different halos (two-halo term). For a detailed description for the HOD model we employ here, see Appendix A.

First, consider the case when $P_{\text {sat }}\left(m_{*} \mid m_{\text {sub }}\right)=$ $P_{\text {cen }}\left(M_{*} \mid M_{\mathrm{h}}\right)$. The short-dashed curves in Fig. 4 show the projected correlation functions in five stellar mass bins for the case the $m_{\text {sub }}^{\text {obs }}$ subhCMF was used. The Yang et al. (2011) galaxy projected correlation functions from the DR7 SDSS are plotted as filled circles with error bars. The resulting correlation functions are clearly below observations, mainly in the one-halo term. This is because using $m_{\text {sub }}^{\text {obs }}$ underestimates the satellite GSMF and CSMF, resulting in a strong deficit of satellite galaxies. Observe that if $N_{s} \sim 0$, then $N \sim N_{c}$ and therefore, $b_{g}\left(M_{*}\right) \sim\left\langle b\left(M_{\mathrm{h}}\right)\right\rangle$ where $\left\langle b\left(M_{\mathrm{h}}\right)\right\rangle$ is the mean weighted halo bias function, see Eq. A5.

When using the subhCMF for $m_{\text {sub }}^{\text {acc }}$ instead of $m_{\text {sub }}^{\text {obs }}$ (dot-short-dashed curves), the agreement with the observed correlation functions is better, though at scales where the one-halo term dominates, the predictions are still below observations. This is, again, because the satellite GSMF and CSMF are underestimated in this case. We remark that using the (average or total) SHMR obtained with the standard AMT in the HOD model by matching the total GSMF to the total halo+subhalo mass function (in the case of $m_{\mathrm{sub}}^{\mathrm{acc}}$ ), leads to excellent agreement with the observed correlation functions, a result that is well known. However in this case the SSMR is not constrained, instead it is implicitly assumed to be equal to the central SHMR (for $m_{\text {sub }}^{\text {acc }}$ ). With our extended AMT, we can explicitly separate both mass relations. When they are assumed to be equal and the central SHMR is constrained with the central GSMF, then we obtain the predictions already shown, in particular the correlation functions. The fact that the predicted correlation functions, when $m_{\mathrm{sub}}^{\mathrm{acc}}$ is used, are close to those predicted in the standard AMT (and to the observed ones) is because the central and average SHMR are indeed close, as we discussed in $\S \S 3.1$, see Fig. 2 .

Thus, under the assumption that $P_{\text {sat }}\left(m_{*} \mid m_{\text {sub }}\right)=$ $P_{\text {cen }}\left(M_{*} \mid M_{\mathrm{h}}\right)$, the observed clustering of galaxies is better reproduced when the subhalo mass in abundance matching is defined as $m_{\text {sub }}^{\text {acc }}$ rather than $m_{\text {sub }}^{\text {obs }}$. Nevertheless, even in the former case, the agreement with observations is only marginal.

We now turn the analysis to the cases where the SSMR is not assumed to be equal to the central SHMR. The black solid and blue long-dashed lines in Fig. 4 show the predicted correlation functions in the cases where either $m_{\text {sub }}$ or $m_{\text {sub }}^{\text {acc }}$ were used. Both cases lead to very similar results and agree very well with observations.

Therefore, the HOD model combined with the central and satellite mass relations independently constrained with the extended AMT, is able to reproduce the observed correlation functions, no matter if $m_{\mathrm{sub}}^{\mathrm{obs}}$ or $m_{\mathrm{sub}}^{\mathrm{acc}}$ are used. This successful prediction is a consequence of the good agreement obtained between our predicted satellite CSMFs and those inferred from observations ( $\S \S 3.2$ and Fig. 3).

\section{SUMMARY AND DISCUSSION}




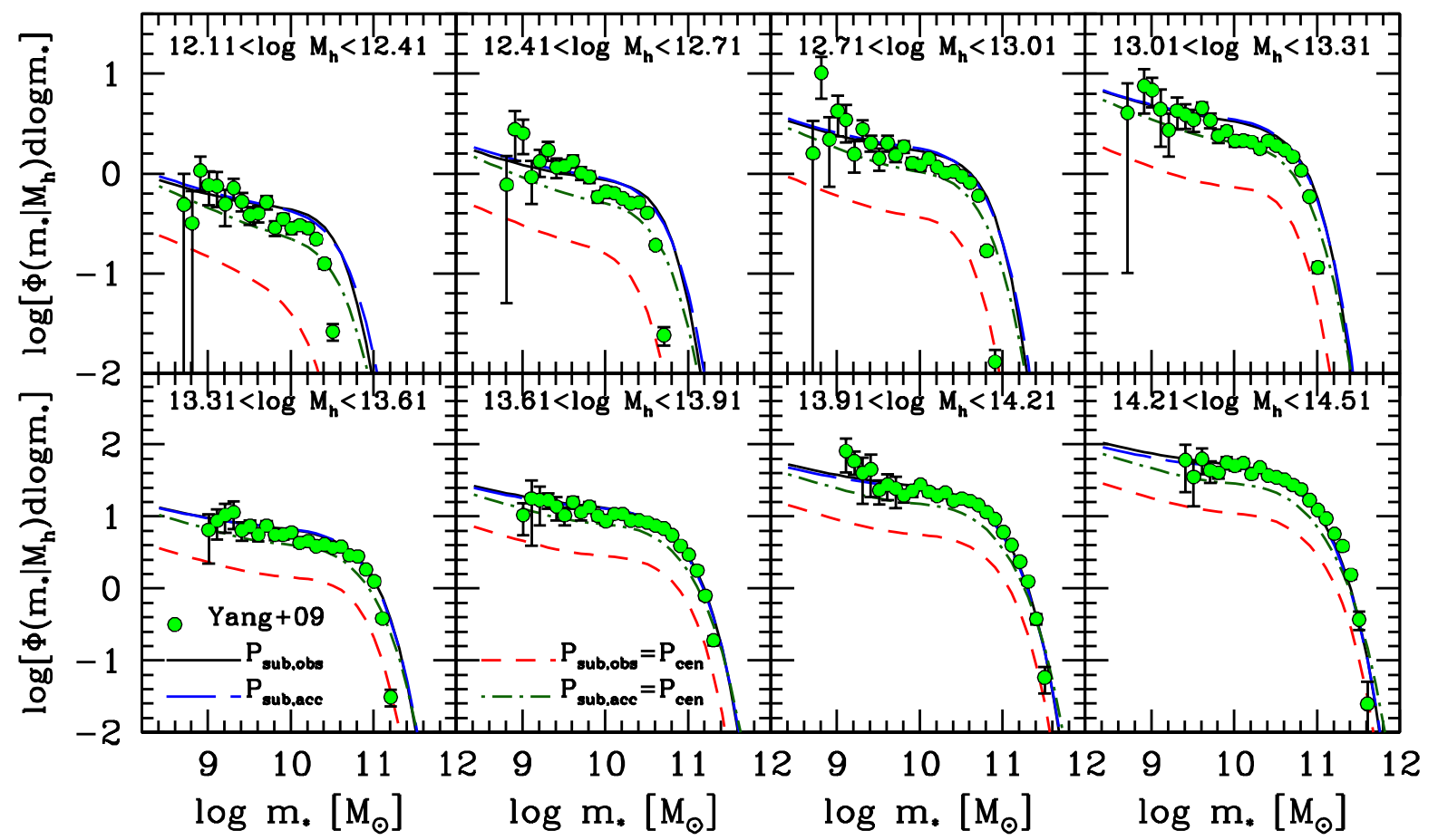

FIG. 3.- Density-weighted average satellite CSMF in eight halo mass intervals. Red short-dashed and green short-dashed-dot lines are for the cases when the central SHMR and the SSMR were assumed to be equal and $m_{\mathrm{sub}}^{\text {obs }}$ and $m_{\mathrm{sub}}^{\text {acc }}$ were used, respectively. The black solid and blue long-dashed lines are again for $m_{\mathrm{sub}}^{\mathrm{obs}}$ and $m_{\mathrm{sub}}^{\mathrm{acc}}$, respectively, but in the case the central and satellite mass functions were independently constrained by means of our extended AMT (they overlap most of time). Filled circles with error bars show the CSMFs inferred from observations by YMB09. Note that their halo masses were converted to match our virial definition.

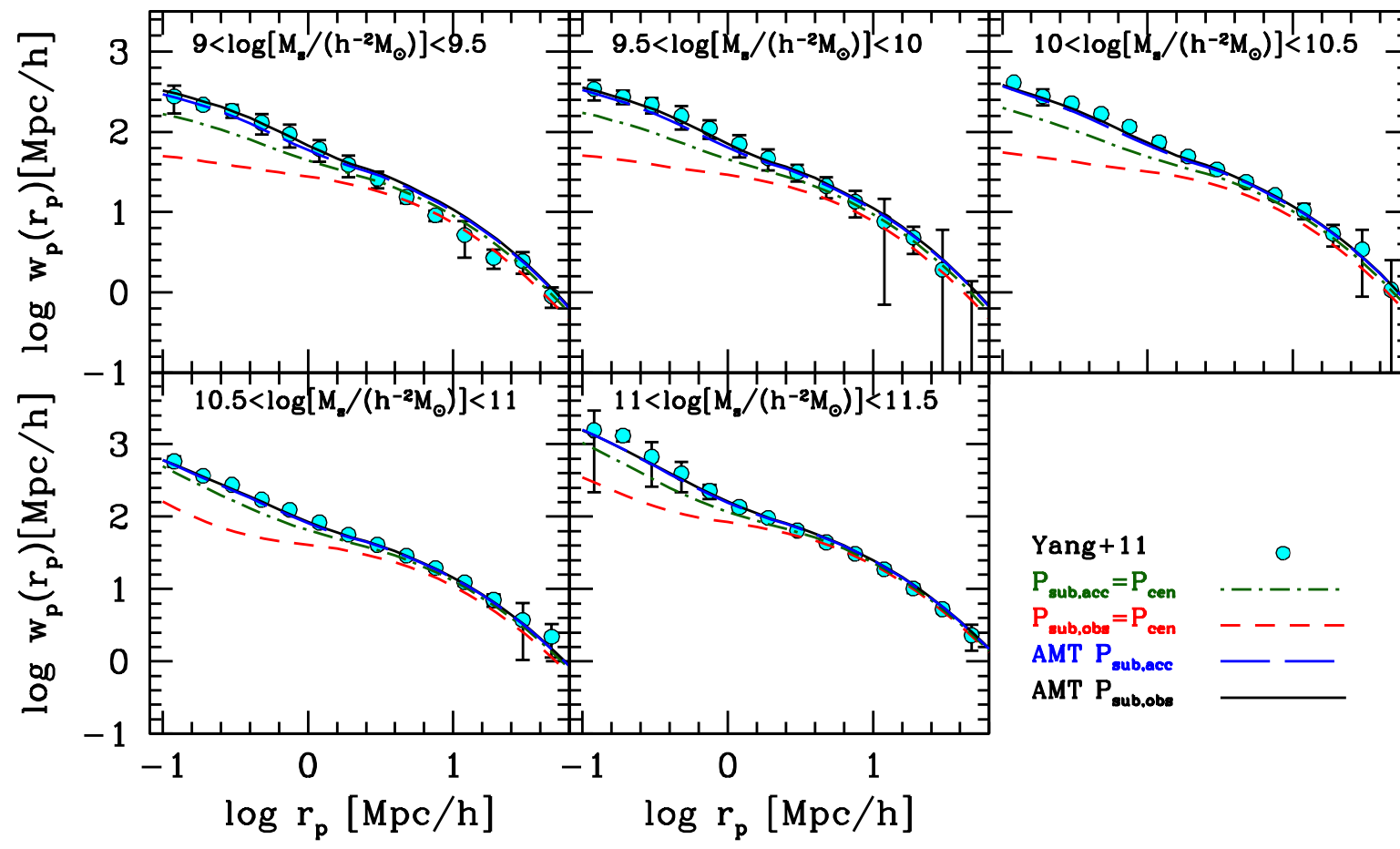

FIG. 4.- Projected two-point correlation functions of galaxies in five stellar mass intervals obtained with the HOD model using different galaxy-halo mass relations obtained with our AMT. Gray short-dashed and green short-dashed-dot lines are for the cases when the central SHMR and the SSMR were assumed to be equal and $m_{\mathrm{sub}}^{\mathrm{obs}}$ and $m_{\mathrm{sub}}^{\text {acc }}$ were used, respectively. The black solid and blue long-dashed lines are again for $m_{\mathrm{sub}}^{\mathrm{obs}}$ and $m_{\mathrm{sub}}^{\mathrm{acc}}$, respectively, but in the case the central and satellite mass functions were independently constrained (they overlap most of time). The observed projected correlation functions reported in Yang et al. (2011) are shown by filled circles with error bars. 
In this paper, we extend the AMT in order to constrain both the central stellar-halo and the satellitesubhalo mass relations separately, using as an input (i) the distinct halo and subhalo mass functions, and (ii) the observed central and satellite GSMFs. Our formalism, by construction, predicts also the satellite CSMFs as a function of host halo mass, and when applied to a HOD model, allows to predict the spatial correlation functions. We present results for the cases when the SSMR is assumed to be equal to the central SHMR, $P_{\text {sat }}\left(m_{*} \mid m_{\text {sub }}\right)=P_{\text {cen }}\left(M_{*} \mid M_{\mathrm{h}}\right)$, and when both mass relations are constrained independently (i.e., it is not assumed that $\left.P_{\text {sat }}\left(m_{*} \mid m_{\text {sub }}\right)=P_{\text {cen }}\left(M_{*} \mid M_{\mathrm{h}}\right)\right)$. All our analysis is carried out for subhalo masses defined at accretion time, $m_{\text {sub }}^{\text {acc }}$, and at the observed time (present day), $m_{\text {sub }}^{\text {obs }}$. The main results and conclusions are as follows:

- Assuming that the mass relation between satellites and subhalos is identical to the mass relation between centrals and distinct halos (including their intrinsic scatters $), \quad P_{\text {sat }}\left(m_{*} \mid m_{\text {sub }}\right)=P_{\text {cen }}\left(M_{*} \mid M_{\mathrm{h}}\right)$, the predicted satellite GSMF, CSMFs and projected two-point correlation functions lie below those obtained from observations for both definitions of $m_{\mathrm{sub}}$, though the disagreements are small when $m_{\mathrm{sub}}=m_{\mathrm{sub}}^{\mathrm{acc}}$ (Figs. 1, 3, 4). We conclude that assuming $P_{\text {sat }}\left(m_{*} \mid m_{\text {sub }}\right)=P_{\text {cen }}\left(M_{*} \mid M_{\mathrm{h}}\right)$ leads to predictions in disagreement with observations, specially when $m_{\text {sub }}^{\text {obs }}$ is used.

- When the SSMR is no longer assumed to be equal to the central SHMR and instead is constrained by means of the observed satellite GSMF, the predicted satellite CSMFs and projected correlation functions agree in general with observations, both for $m_{\mathrm{sub}}^{\mathrm{obs}}$ and $m_{\mathrm{sub}}^{\mathrm{acc}}$. However, for halo masses lower than $\sim 10^{13} \mathrm{M}_{\odot}$, the number of very massive (rare) satellites is over-predicted.

- The resulting $m_{\mathrm{sub}}-m_{*}$ relations when using either $m_{\text {sub }}^{\text {obs }}$ or $m_{\text {sub }}^{\text {acc }}$ are quite different from each other, and in each case are different from the central SHMR (Fig. 2). For a given stellar mass, the satellite subhalo mass is smaller than central halo mass, and the mass difference is increasing the lower the mass is. These differences are dramatic when $m_{\text {sub }}^{\text {obs }}$ is used.

- Our density-weighted average (centrals + satellites) SHMRs are close to the central SHMR when either $m_{\mathrm{sub}}^{\mathrm{obs}}$ or $m_{\mathrm{sub}}^{\mathrm{acc}}$ is used (central galaxies dominate in the YMB09 catalog). Such an average SHMR coincides conceptually with the one inferred from matching the total (centrals + satellites) cumulative GSMF and the halo + subhalo cumulative mass function (standard AMT).

\subsection{On the inference of the SSMR and its implications for the average mass relation}

The conclusions listed above can be well understood by examining the basic ideas behind the extended AMT, as we show in Section 2.2. Essentially, matching abundances of satellite galaxies to subhalos is equivalent to matching their corresponding occupational numbers, that is:

$$
\left\langle N_{s}\left(>m_{*} \mid M_{\mathrm{h}}\right)\right\rangle=\left\langle N_{\mathrm{sub}}\left(>m_{\mathrm{sub}} \mid M_{\mathrm{h}}\right)\right\rangle .
$$

The opposite is also true: matching their corresponding occupational numbers is equivalent to matching their abundances. This is an important result because it shows that once $P_{\text {sat }}\left(m_{*} \mid m_{\text {sub }}\right.$ ) (and $P_{\text {cen }}\left(M_{*} \mid M_{\mathrm{h}}\right)$ ) is properly constrained, we will obtain the correct conditional mass functions and consequently the correct spatial clustering for galaxies.

The above means that there is a unique $m_{\mathrm{sub}}\left(m_{*}\right)$ relationship for each definition of $m_{\text {sub }}$, which depends solely on $\left\langle N_{\text {sub }}\left(>m_{\text {sub }} \mid M_{\mathrm{h}}\right)\right\rangle$. Because of this uniqueness, it follows that the $m_{\mathrm{sub}}^{\mathrm{obs}}\left(m_{*}\right)$ and $m_{\mathrm{sub}}^{\mathrm{acc}}\left(m_{*}\right)$ relations should be different, and any incorrect assumption on each one of these relations will lead to inconsistencies in the conditional mass functions and spatial clustering of galaxies, as for example those that we have found here when $P_{\text {sat }}\left(m_{*} \mid m_{\text {sub }}\right)$ was assumed to be equal to $P_{\text {cen }}\left(M_{*} \mid M_{\mathrm{h}}\right)$. Under this assumption, when $m_{\text {sub }}^{\text {acc }}$ was used, the inconsistencies were actually small. This is because in this case the "incorrect" assumption for the SSMR is actually not too far from the "correct" result obtained when $P_{\text {sat }}\left(m_{*} \mid m_{\text {sub }}^{\text {obs }}\right)$ is independently constrained (compare dot-dashed and solid green curves in Fig. 2), contrary to what happens when $m_{\text {sub }}^{\text {obs }}$ is used.

It is important to remark that in the standard AMT, only the average SHMR is constrained (using the total GSMF), leaving unconstrained the SSMR, something that on its own introduces a large uncertainty in the average SHMR (see Neistein et al. 2011). We have shown that such average SHMR is conceptually equal to the density-weighted average mass relation obtained here from the observationally constrained central SHMR and SSMR. Therefore, our resulting average mass relation is expected to be less uncertain than previous determinations. On the other hand, this average mass relation is expected to be close to the central SHMR because most of the galaxies in the used observational catalog are centrals.

We conclude that in order to properly infer the SSMR and the central SHMR at the same time, and this way reduce the uncertainty in the average SHMR, more observational constraints than the total GSMF are necessary. The most obvious and direct is the GSMF decomposed into central and satellite galaxies, something that was provided by YMB09. However, observe that, according to eq. (11), the satellite CSMFs or the clustering of galaxies, modulo the observational errors, provide observational constraints that lead to similar inferences of the SSMR, because of the uniqueness of this relation for a given well defined $\left\langle N_{\text {sub }}\left(>m_{\text {sub }} \mid M_{\mathrm{h}}\right)\right\rangle$ (see above).

Finally, we note that obtaining the SSMR for the subhalo mass defined at the accretion time introduces uncertainties due to our ignorance about evolutionary processes of the stellar mass since accretion. This does not happen when the SSMR is obtained for both the satellite and subhalo masses defined at the same epoch, for instance the present time. When matching abundances for the $m_{\text {sub }}^{\text {acc }}$ case, the fact that (1) $m_{\text {sub }}^{\text {acc }}$ is itself a cumulative distribution of all objects accreted over a period of time, and that (2) $m_{*}$ may have changed be- 
tween accretion and observation, are not taken into account. In other words, it is implicitly assumed that the satellite stellar mass stops evolving soon after accretion. In reality the situation is actually quite complex in the sense that, depending on the accretion time and the orbit of the satellites, the evolution of their stellar masses is diverse, with some of them early quenched and others actively evolving, perhaps in some cases as the central ones of the same mass (see e.g., Wetzel. Tinker \& Conroy 2011, for semi-empirical inferences on such a complexity of galaxy evolution in groups). This diversity introduces an intrinsic uncertainty on the results. Such an uncertainty might be accounted for the probability distribution functions: $P\left(m_{*} \mid m_{*, \text { acc }}, z\right)$, which gives the probability that a satellite accreted at epoch $z$ evolves, on average, to the observed satellite $m_{*}$, and $P\left(m_{*, \text { acc }} \mid m_{\text {sub }}^{\text {acc }}, z\right)$, which gives the probability that a subhalo $m_{\mathrm{sub}}^{\text {acc }}$ hosts a galaxy of mass $m_{* \text {,acc }}$ at the time of accretion. Now, the satellite CSMF (Eq. 2) can be written as (Mo, van den Bosch \& White 2010):

$$
\begin{array}{r}
\Phi_{s}\left(m_{*} \mid M_{\mathrm{h}}\right)=\iiint P\left(m_{*} \mid m_{*, \mathrm{acc}}, z\right) P\left(m_{*, \mathrm{acc}} \mid m_{\mathrm{sub}}^{\mathrm{acc}}, z\right) \times \\
\Phi\left(m_{\mathrm{sub}}^{\mathrm{acc}} \mid M_{\mathrm{h}}, z\right) d m_{*, \mathrm{acc}} d m_{\mathrm{sub}}^{\mathrm{acc}} d z .
\end{array}
$$

Note that in our analysis in $\S \S 3.1$, we implicitly assume that the stellar mass of satellite galaxies does not change once they become satellites, i.e. $P\left(m_{*} \mid m_{*, \text { acc }}, z\right)=$ $\delta\left(m_{*}-m_{*, \text { acc }}, z\right)$, and that $P\left(m_{*, \text { acc }} \mid m_{\text {sub }}^{\text {acc }}, z\right)$ is independent of redshift. Thus, the application of the AMT to infer the satellite CSMF and the $m_{*}-m_{\text {sub }}$ relation for subhalo mass defined at its accretion time formally requires more observational constraints at higher redshifts. This is a problem already faced by previous authors (e.g., Yang et al. 2011).

The above is not the only way to formally write the satellite CSMF; it can be written in a way that instead of implying knowledge of the change of $m_{*}$ from accretion to observation, implies just knowledge on the change of the subhCMF between these two epochs. Let us consider the distribution function, $P_{\mathrm{acc}}\left(m_{\mathrm{sub}}^{\mathrm{obs}} \mid m_{\mathrm{sub}}^{\mathrm{acc}}, z\right)$, giving the probability that halos accreted at epoch $z$ evolve, on average, to the observed (present-day) subhalos $m_{\mathrm{sub}}^{\mathrm{obs}}$, and the probability distribution function of these subhalos of hosting a galaxy of mass $m_{*}, P_{\text {sat }}\left(m_{*} \mid m_{\text {sub }}^{\text {obs }}\right)$. In this case, the satellite CSMF (Eq. 2) is written as

$$
\begin{array}{r}
\Phi_{s}\left(m_{*} \mid M_{\mathrm{h}}\right)=\iiint P_{\mathrm{sat}}\left(m_{*} \mid m_{\mathrm{sub}}^{\mathrm{obs}}\right) P_{\mathrm{acc}}\left(m_{\mathrm{sub}}^{\mathrm{obs}} \mid m_{\mathrm{sub}}^{\mathrm{acc}}, z\right) \times \\
\Phi\left(m_{\mathrm{sub}}^{\mathrm{acc}} \mid M_{\mathrm{h}}, z\right) d m_{\mathrm{sub}}^{\mathrm{obs}} d m_{\mathrm{sub}}^{\mathrm{acc}} d z,
\end{array}
$$

and therefore the satellite GSMF, Eq. 4, is given by

$$
\begin{array}{r}
\phi_{\mathrm{sat}}\left(m_{*}\right)=\iiint P_{\mathrm{sat}}\left(m_{*} \mid m_{\mathrm{sub}}^{\mathrm{obs}}\right) P_{\mathrm{acc}}\left(m_{\mathrm{sub}}^{\mathrm{obs}} \mid m_{\mathrm{sub}}^{\mathrm{acc}}, z\right) \times \\
\phi_{\mathrm{sub}}\left(m_{\mathrm{sub}}^{\mathrm{acc}}, z\right) d m_{\mathrm{sub}}^{\mathrm{obs}} d m_{\mathrm{sub}}^{\mathrm{acc}} d z .
\end{array}
$$

Since the $m_{\text {sub }}^{\text {acc }}$ subHMF would evolve into the $m_{\text {sub }}^{\text {obs }}$
subHMF, we write

$\phi_{\mathrm{sub}}\left(m_{\mathrm{sub}}^{\mathrm{obs}}\right)=\iint P_{\mathrm{acc}}\left(m_{\mathrm{sub}}^{\mathrm{obs}} \mid m_{\mathrm{sub}}^{\mathrm{acc}}, z\right) \phi_{\mathrm{sub}}\left(m_{\mathrm{sub}}^{\mathrm{acc}}, z\right) d m_{\mathrm{sub}}^{\mathrm{acc}} d z$.

This last equation is the abundance matching of accreted subhalos to present-day subhalos. Therefore,

$$
\phi_{\text {sat }}\left(m_{*}\right)=\int P_{\text {sat }}\left(m_{*} \mid m_{\mathrm{sub}}^{\mathrm{obs}}\right) \phi_{\mathrm{sub}}\left(m_{\mathrm{sub}}^{\mathrm{obs}}\right) d m_{\mathrm{sub}}^{\mathrm{obs}} .
$$

This equation is nothing but abundance matching satellite galaxies to subhalos at the time they are observed. Hence, the reason that the satellite GSMF matches the subHMF in a more direct way for subhalo masses defined at the observation time (eq. 16) than at the accretion time (eq. 12), is that in the latter case the unknown $P\left(m_{*} \mid m_{*, \text { acc }}, z\right)$ and $P\left(m_{*, \text { acc }} \mid m_{\text {sub }}^{\text {acc }}, z\right)$ "evolutionary" functions have to be introduced. However, we acknowledge that for the former case, our ignorance on the scatter around the SSMR is a also potential source of uncertainty. All our calculations are under the assumption that this scatter is the same as the scatter of the central SHMR. In any case, even if these scatters are different, note that including scatter affects the stellar-to(sub)halo mass relation only at its high-mass end, where on average satellites are expected to be accreted recently, hence their SSMR and scatter are yet similar to those of centrals/halos.

\subsection{On the intrinsic scatter in the SSMR}

A possible source of systematic errors in our analysis is the assumption that the intrinsic scatter around the $\mathrm{SSMR}, \sigma_{s}$ is constant and equal to the scatter around the central SHMR. To probe the impact of this assumption we repeated all our analysis but this time assuming $\sigma_{s}=0$. When comparing the results using $\sigma_{s}=0$ to those obtained based on $\sigma_{s}=0.173$ dex, we find that they are consistent with each other, and therefore with the satellite CSMFs and with the galaxy spatial clustering measured from the YMB09 catalog. In more detail, we find that the resulting CSMF's reproduce observations for $\sigma_{s}=0$ slightly better than for $\sigma_{s}=0.173 \mathrm{dex}$, especially at the massive end. This is because when the intrinsic scatter is not taken into account $\left(\sigma_{s}=0\right)$, the shape of the SSMRs steepens at the massive end (see also Behroozi. Conrov \& Wechsler 2010). Consequently, for a given $m_{*}$, the subhalo mass is larger, and the abundances of larger (sub)halos is lower in general than those of smaller halos. Therefore, the number density of satellites at the massive end is lower. However, the projected correlation functions remain almost the same because the probability of finding a massive satellite galaxy in host halos less massive than $\log M_{\mathrm{h}} \sim 13$ is very low. They do not contribute significantly to the mean total density of galaxies. Although better models are needed in order to give a realistic form for $\sigma_{s}$, our main conclusions seem to be robust to variations in the adopted value for $\sigma_{s}$.

\subsection{Implications for satellite/subhalo evolution}

The local SSMR obtained for both definitions of the subhalo mass, $m_{\text {sub }}^{\text {acc }}$ and $m_{\text {sub }}^{\text {obs }}$, are such that at halos masses smaller than $2-10 \times 10^{13} \mathrm{M}_{\odot}$ and at a given galaxy stellar mass, the corresponding subhalo mass is 
smaller on average than the halo mass of centrals (Fig. 2). This difference increases the smaller the mass is, and much more for the subhalo mass defined at the observed time (present-day). In the case of $m_{\mathrm{sub}}^{\mathrm{acc}}$, the differences might be because the halo mass at the epoch it became a subhalo (accretion time) is smaller than its present-day counterpart at a given stellar mass and/or because the satellite stellar mass increased faster than the central one for a given halo mass. In fact, it is difficult to make any inference in this case because the abundance matching is between local galaxies and (sub)halos at different past epochs. In any case, the fact that the inferred mass relations for satellites and centrals when $m_{\text {sub }}^{\text {acc }}$ is used are not too different, suggests that the central galaxy-distinct halo mass relation does not change too much with time, at least since the epoch at which most of the subhalos were accreted.

When $m_{\text {sub }}^{\text {obs }}$ is used, both abundances of satellites and subhalos are matched at the same epoch, the observation (present-day) time. In this case the strong difference between the satellite and central mass relations can be interpreted mainly as the result of subhalo mass loss due to tidal stripping. Besides, the smaller the subhalo, the larger is the mass loss on average. Probably, the different evolution in stellar mass between central and satellite galaxies could also play a role for the differences but not as significant a role the one related to halo and subhalo mass evolution.

From Fig. 22 one sees that for a given $m_{*}$, the $m_{\mathrm{sub}}^{\mathrm{obs}}$ to $-m_{\text {sub }}^{\text {acc }}$ ratio is $0.35-0.40$ for the smallest masses up to $m_{*} \sim 2 \times 10^{11} \mathrm{M}_{\odot}$. At larger masses, this ratio rapidly tends to 1 . Therefore, the subhalos of satellites galaxies less massive than $m_{*} \sim 2 \times 10^{11} \mathrm{M}_{\odot}$ have lost, on average (for all host halo masses 4 ), $65-60 \%$ of their masses since they were accreted. It should be noted that this is a rough approximation and the evolution of the stellar mass since the satellite was accreted should be taken into account, see $\S 4.1$. This above result shows us that the galaxy-(sub)halo connection for satellite galaxies is far from direct; present-day satellites of masses $m_{*} \sim 7 \times 10^{8}$ $\mathrm{M}_{\odot}$ and larger have formed in subhalos that at the time they were accreted onto galaxy sized halos were on average a factor 2.5-3 larger than at the present epoch. This has severe implications for studies aimed to constrain the $\Lambda$-CDM scenario at the level of subhalo/satellite distributions.

For example, it has been discussed that seeding the subhalos in simulations of MW-like halos by using an extrapolation to low masses of the stellar-halo mass relation obtained by means of the AMT, predicts a MW dwarf spheroidal (dSph) luminosity function in agreement with the observed one. However, the circular velocities at the maximum (or the masses at the infall) of the subhalos associated to the dSphs result significantly larger than inferences from observed kinematics (Boylan-Kolchin, Bullock \& Kaplinghat 2011).

In the right panel of Fig. 2 we have plotted the extrapolation to low masses of our SSMRs, both for subhalo masses defined at the present day (red line) and at the infall time (blue line). The observational points in the panel are for MW satellites, which subhalo masses were estimated at their truncation radii. Thus, if we assume that these masses are roughly equal to the present-day subhalo masses in the $\Lambda$-CDM simulations, then the simulated subhalo masses, $m_{\text {sub }}^{\text {obs }}$, are up to $\approx 10-30$ times larger than those associated to $\mathrm{dSphs.} \mathrm{If} \mathrm{the} \mathrm{comparison} \mathrm{is} \mathrm{done} \mathrm{with} \mathrm{the} \mathrm{extrapolation}$ of the average (or central) SHMR, then the differences increases by a factor of $\sim 3$ more (see also Fig. 7 in Boylan-Kolchin, Bullock \& Kaplinghat 2011).

Our extrapolated results show that the discrepancy in subhalo mass between MW bright dSphs and $\Lambda$-CDM simulations is smaller than previously reported but is still significant. Note that for the extrapolation, we have used the same slope of the YMB09 satellite GSMF at the low mass end, $\alpha=-1.25$ (Fig. 1). If this slope steepens for smaller masses, for example to a value of $\alpha=-1.6$,

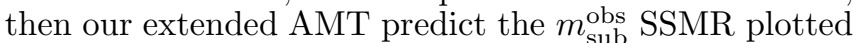
as the gray dotted-dashed curve in Fig. 2, which is already consistent with the dynamical estimates.

The GSMF at low masses may be significantly incomplete because of missing low-surface brightness galaxies. By taking into account the bivariate distribution of stellar mass versus surface brightness, Baldry, Glazebrook \& Driver (2008) have found evidence for an upturn in the faint-end GSMF slope $(\alpha \approx$ $-1.6)$ for a subsample of field SDSS galaxies. More recently, using the GAMA survey, a slope of $\alpha \approx-1.47$ has been reported (Baldry et al. 2011). Steep faint-end slopes have been also found at higher redshifts. For instance, using the COSMOS field, Drory et al. (2009) have measured slopes of $\alpha \sim-1.7$ at all redshifts $z \leq 1$ There are also pieces of evidence that the faint-end slope of the GSMF (or luminosity function) changes with the environment: in clusters of galaxies it steepens significantly (for a discussion see Baldry. Glazebrook \& Driver 2008, and the references therein). The cluster GSMF is actually related to the satellite GSMF, through the satellite CSMF.

We conclude that using a correct AMT for connecting satellite galaxies to their present-day subhalos and assuming a steep faint-end slope in the satellite GSMF $(\alpha \sim-1.6)$, the predicted SSMR for the $\Lambda$-CDM cosmogony would be consistent with the dynamics of $\mathrm{MW}$ satellites.

We thank Alexie Leathaud, Ramin Skibba, Surhud More, Xiaohu Yang, and the anonymous Referee for useful comments, and suggestions. A. R-P acknowledges a graduate student fellowship provided by CONACyT. N. D. and V. A. acknowledge to CONACyT grant 128556 (Ciencia Básica). V.A acknowledges PAPIIT-UNAM grant IN114509. We are grateful to X. Yang for providing us in electronic form their data for the CSMFs.

\section{REFERENCES}

\footnotetext{
4 The dependence of the satellite subhalo mass loss on host halo mass will be explored elsewhere.
}

Angulo R. E., Lacey C. G., Baugh C. M., Frenk C. S., 2009, MNRAS, 399, 983

Avila-Reese V., Colín P., González-Samaniego A., Valenzuela O.,

Firmani C., Velázquez H., Ceverino D., 2011, ApJ, 736, 134 
Baldry I. K. et al., 2011, ArXiv e-prints

Baldry I. K., Glazebrook K., Driver S. P., 2008, MNRAS, 388, 945

Behroozi P. S., Conroy C., Wechsler R. H., 2010, ApJ, 717, 379

Berlind A. A., Weinberg D. H., 2002, ApJ, 575, 587

Blanton M. R., Geha M., West A. A., 2008, ApJ, 682, 861

Boylan-Kolchin M., Bullock J. S., Kaplinghat M., 2011, ArXiv e-prints

Boylan-Kolchin M., Springel V., White S. D. M., Jenkins A., 2010, MNRAS, 406, 896

Chabrier G., 2003, PASP, 115, 763

Conroy C., Wechsler R. H., 2009, ApJ, 696, 620

Conroy C., Wechsler R. H., Kravtsov A. V., 2006, ApJ, 647, 201

Cooray A., Sheth R., 2002, PhR, 372, 1

Cuesta A. J., Prada F., Klypin A., Moles M., 2008, MNRAS, 389 , 385

Drory N. et al., 2009, ApJ, 707, 1595

Firmani C., Avila-Reese V., 2010, ApJ, 723, 755

Firmani C., Avila-Reese V., Rodríguez-Puebla A., 2010, MNRAS, 404, 1100

Gao L., Frenk C. S., Boylan-Kolchin M., Jenkins A., Springel V., White S. D. M., 2011, MNRAS, 410, 2309

Gao L., White S. D. M., Jenkins A., Stoehr F., Springel V., 2004, MNRAS, 355, 819

Giocoli C., Tormen G., Sheth R. K., van den Bosch F. C., 2010, MNRAS, 404, 502

Giocoli C., Tormen G., van den Bosch F. C., 2008, MNRAS, 386, 2135

Guo Q., White S., Li C., Boylan-Kolchin M., 2010, MNRAS, 404, 1111

Klypin A. A., Trujillo-Gomez S., Primack J., 2011, ApJ, 740, 102

Kravtsov A. V., Berlind A. A., Wechsler R. H., Klypin A. A., Gottlöber S., Allgood B., Primack J. R., 2004, ApJ, 609, 35

Leauthaud A., Tinker J., Behroozi P. S., Busha M. T., Wechsler R. H., 2011a, ApJ, 738, 45

Leauthaud A. et al., 2011b, ArXiv e-prints

Mandelbaum R., Seljak U., Kauffmann G., Hirata C. M., Brinkmann J., 2006, MNRAS, 368, 715

Mo H., van den Bosch F. C., White S., 2010, Galaxy Formation and Evolution, Mo, H., van den Bosch, F. C., \& White, S., ed.

More S., van den Bosch F. C., Cacciato M., Skibba R., Mo H. J., Yang X., 2011, MNRAS, 410, 210

Moster B. P., Somerville R. S., Maulbetsch C., van den Bosch F. C., Macciò A. V., Naab T., Oser L., 2010, ApJ, 710, 903 Muñoz-Cuartas J. C., Macciò A. V., Gottlöber S., Dutton A. A., 2011, MNRAS, 411, 584

Navarro J. F., Frenk C. S., White S. D. M., 1997, ApJ, 490, 493
Neistein E., Li C., Khochfar S., Weinmann S. M., Shankar F., Boylan-Kolchin M., 2011, MNRAS, 416, 1486

Press W. H., Teukolsky S. A., Vetterling W. T., Flannery B. P., 1992, Numerical recipes in C. The art of scientific computing, Press, W. H., Teukolsky, S. A., Vetterling, W. T., \& Flannery, B. P. , ed.

Rodríguez-Puebla A., Avila-Reese V., Firmani C., Colín P., 2011, RevMxAA, 47, 235

Shankar F., Lapi A., Salucci P., De Zotti G., Danese L., 2006 , ApJ, 643, 14

Sheth R. K., Mo H. J., Tormen G., 2001, MNRAS, 323, 1

Sheth R. K., Tormen G., 1999, MNRAS, 308, 119

Skibba R. A., van den Bosch F. C., Yang X., More S., Mo H., Fontanot F., 2011, MNRAS, 410, 417

Smith R. E. et al., 2003, MNRAS, 341, 1311

Strigari L. E., Bullock J. S., Kaplinghat M., Diemand J., Kuhlen M., Madau P., 2007, ApJ, 669, 676

Strigari L. E., Frenk C. S., White S. D. M., 2010, MNRAS, 408, 2364

Tinker J. L., Weinberg D. H., Zheng Z., Zehavi I., 2005, ApJ, 631,41

Trujillo-Gomez S., Klypin A., Primack J., Romanowsky A. J., 2011, ApJ, 742, 16

Vale A., Ostriker J. P., 2004, MNRAS, 353, 189

van den Bosch F. C., Aquino D., Yang X., Mo H. J., Pasquali A., McIntosh D. H., Weinmann S. M., Kang X., 2008, MNRAS, 387,79

van den Bosch F. C., Tormen G., Giocoli C., 2005, MNRAS, 359, 1029

Weinberg M. D., 1999, in Astronomical Society of the Pacific Conference Series, Vol. 165, The Third Stromlo Symposium:

The Galactic Halo, B. K. Gibson, R. S. Axelrod, \&

M. E. Putman, ed., p. 100

Wetzel A. R., Tinker J. L., Conroy C., 2011, ArXiv e-prints

Yang X., Mo H. J., van den Bosch F. C., 2003, MNRAS, 339, 1057

Yang X., Mo H. J., van den Bosch F. C., 2009a, ApJ, 695, 900

Yang X., Mo H. J., van den Bosch F. C., 2009b, ApJ, 693, 830

Yang X., Mo H. J., van den Bosch F. C., Pasquali A., Li C., Barden M., 2007, ApJ, 671, 153

Yang X., Mo H. J., van den Bosch F. C., Zhang Y., Han J., 2011, ArXiv e-prints

Zehavi I. et al., 2011, ApJ, 736, 59

Zehavi I. et al., 2005, ApJ, 630, 1

Zentner A. R., Berlind A. A., Bullock J. S., Kravtsov A. V., Wechsler R. H., 2005, ApJ, 624, 505

Zentner A. R., Bullock J. S., 2003, ApJ, 598, 49

Zheng Z. et al., 2005, ApJ, 633, 791

\section{APPENDIX}

\section{THE SPATIAL CLUSTERING OF GALAXIES IN THE HOD MODEL}

Here we review the main ideas used to infer the spatial clustering of galaxies based on a HOD model. We assume that the most massive galaxy in terms of stellar mass within a halo of mass $M_{\mathrm{h}}$ is its central galaxy. Consequently the remaining galaxies are all satellites. We let them follow the mass density profile of the host halo. We denote the cumulative number of central and satellite galaxies with stellar masses greater than $M_{*}$ as $N_{c}$ and $N_{s}$, respectively.

The two point correlation function is decomposed into two terms,

$$
1+\xi_{\mathrm{gg}}(r)=\left[1+\xi_{\mathrm{gg}}^{1 \mathrm{~h}}(r)\right]+\left[1+\xi_{\mathrm{gg}}^{2 \mathrm{~h}}(r)\right]
$$

with $1+\xi_{\mathrm{gg}}^{\mathrm{hh}}(r)$ describing galaxy pairs within the same halo (the one-halo term), and $1+\xi_{\mathrm{gg}}^{2 \mathrm{~h}}(r)$ describing the correlation between galaxies occupying different halos (the two-halo term).

We compute the one-halo term as

$$
1+\xi_{\mathrm{gg}}^{\mathrm{h}}(r)=\frac{1}{2 \pi r^{2} n_{g}^{2}} \int_{0}^{\infty} \frac{\langle N(N-1)\rangle}{2} \lambda(r) \phi_{\mathrm{h}}\left(M_{\mathrm{h}}\right) d M_{\mathrm{h}},
$$

for pairs separated by a distance $r \pm d r / 2$. Here $\langle N(N-1)\rangle / 2$ is the total mean number of galaxy pairs within halos $M_{\mathrm{h}}$ following a pair distribution $\lambda(r) d r$, and a mean number density $n_{g}\left(M_{*}\right)$. The contribution to the total mean number of galaxy pairs from central-satellite pairs and satellite-satellite pairs is

$$
\frac{\langle N(N-1)\rangle}{2} \lambda(r) d r=\left\langle N_{c}\right\rangle\left\langle N_{s}\right\rangle \lambda_{c, s}(r) d r
$$




$$
+\frac{\left\langle N_{s}\left(N_{s}-1\right)\right\rangle}{2} \lambda_{s, s}(r) d r
$$

As commonly assumed in HOD models, the number of central-satellite pairs follow the normalized mass density halo profile, taken to be of Navarro, Frenk \& White (1997) shape. The number of satellite-satellite pairs is then related to the normalized density profile convolved with itself.

Halo profiles are defined in terms of the total halo mass and the concentration parameter. We use the relation between concentration parameter $c_{\mathrm{NFW}}$ and halo mass obtained by Muñoz-Cuartas et al. (2011) from fits to $N$-body simulations.

Based on results of high-resolution $N$-body (Kravtsov et al. 2004) and hydrodynamic simulations of galaxy formation (Zheng et al. 2005), we model the second moment of satellite galaxies, $\left\langle N_{s}\left(N_{s}-1\right)\right\rangle$, as a Poisson distribution with mean $\left\langle N_{s}\right\rangle^{2}=\left\langle N_{s}\left(N_{s}-1\right)\right\rangle$.

We compute the two-halo term as

$$
\xi_{\mathrm{gg}}^{2 \mathrm{~h}}(r)=b_{g}^{2} \zeta^{2}(r) \xi_{m}(r)
$$

where $\xi_{m}(r)$ is the non-linear matter correlation function (Smith et al. 2003), $\zeta(r)$ is the scale dependence of dark matter halo bias (Tinker et al. 2005), and

$$
b_{g}=\frac{1}{n_{g}} \int_{0}^{\infty} b\left(M_{\mathrm{h}}\right)\left\langle N\left(>M_{*} \mid M_{\mathrm{h}}\right)\right\rangle \phi_{\mathrm{h}}\left(M_{\mathrm{h}}\right) d M_{\mathrm{h}},
$$

is the galaxy bias with $b\left(M_{\mathrm{h}}\right)$ the halo bias function (Sheth. Mo \& Tormen 2001).

Once we have calculated $\xi_{\mathrm{gg}}(r)$, we relate it to the projected correlation function, $w_{\mathrm{p}}\left(r_{\mathrm{p}}\right)$, integration over the line of sight,

$$
w_{\mathrm{p}}\left(r_{\mathrm{p}}\right)=2 \int_{0}^{\infty} \xi_{\mathrm{gg}}\left(\sqrt{r_{\mathrm{p}}^{2}+x^{2}}\right) d x
$$

